\title{
Formulation Development of Mouth Dissolving Film of Etoricoxib for Pain Management
}

\author{
K. Senthilkumar and C. Vijaya \\ Department of Pharmaceutics, Ultra College of Pharmacy, Madurai, Tamil Nadu 625020, India \\ Correspondence should be addressed to C. Vijaya; srikvijaya@gmail.com
}

Received 31 July 2014; Revised 28 December 2014; Accepted 28 December 2014

Academic Editor: Barbara R. Conway

Copyright ( 2015 K. Senthilkumar and C. Vijaya. This is an open access article distributed under the Creative Commons Attribution License, which permits unrestricted use, distribution, and reproduction in any medium, provided the original work is properly cited.

\begin{abstract}
Etoricoxib is a potent, orally active, and highly selective COX-2 inhibitor that exhibits anti-inflammatory, analgesic, and antipyretic activities. The present research was undertaken to develop mouth dissolving films of etoricoxib to have rapid onset of action. Mouth dissolving film (MDF) is a better alternate to oral disintegrating tablets due to its novelty, ease of use, and the consequent patient compliance. Solubility enhancement and taste masking of etoricoxib were the two challenges solved by formulating druginclusion complex with beta-cyclodextrin (BCD). MDF prepared by solvent casting etoricoxib-BCD complex along with HPMC as film forming polymer was found to possess desirable physicomechanical properties. In vitro release of etoricoxib from MDF in simulated salivary fluid and $0.1 \mathrm{~N} \mathrm{HCl}$ was more than $95 \%$ within 2 minutes. Taste masking and in vivo disintegration were in acceptable range as assessed by human volunteers. Etoricoxib MDF was further characterized by differential scanning calorimetry, powder X-ray diffraction, and scanning electron microscopy. The index of analgesia shown by etoricoxib MDF was comparable to that of immediate release tablets (100\% activity within 40 minutes) in animal studies. Conclusively, the present study documents the development of a commercially viable formula for an MDF of etoricoxib with rapidity in pain management.
\end{abstract}

\section{Introduction}

Rapid or fast dissolving oral thin film is becoming an increasingly popular drug delivery system because of its wide and varied benefits. On contact with saliva, it dissolves within a few seconds, without the need of water, making them particularly suitable for paediatric and geriatric patients. As most of the polymers used in mouth dissolving films (MDFs) are amorphous, dispersion of drug in polymer matrix aids rapid dissolution. These advantages enhance the patient compliance and make pharmaceutical manufacturer invest money in change of the existing products in the market to MDFs [1].

Etoricoxib is a potent, orally active cyclooxygenase- 2 (COX-2) specific inhibitor, indicated for pain and inflammation in osteoarthritis, in rheumatoid arthritis, in acute gouty arthritis, in chronic low back pain, in ankylosing spondylitis, and in other acute and chronic musculoskeletal disorders, primary dysmenorrhoea and pain following dental surgery [2]. Etoricoxib is available as immediate release tablets of 30 ,
60 , or $120 \mathrm{mg}$ [3]. It is a white to off-white powder bitter in taste. Etoricoxib is practically insoluble in water categorized to BCS class II (low soluble, high permeable). Its poor aqueous solubility and dissolution delay the rate of absorption [4]. Formulation of etoricoxib as MDF would improve its aqueous solubility along with fast dissolution of etoricoxib in mouth itself resulting in earlier drug absorption starting from oral cavity itself leading to rapid pain relief.

The main challenges of the present study were taste masking besides improving the aqueous solubility of the drug as medications that enter the oral cavity, irrespective of mode of administration, namely, swallowing and sublingual or oral inhalation, should have an acceptable taste. One of the major barriers that prevents patient from adhering to a prescribed medication regimen has been identified as the unacceptable taste of active pharmaceutical ingredients (APIs) in these dosage forms [5]. Taste has an important role in the development of oral pharmaceuticals, with respect to patient acceptability and compliance, and is one of the prime factors 
determining the market penetration and commercial success of oral formulations, especially in pediatric medicine [6].

Beta-cyclodextrin (BCD) increases the aqueous solubility of many poorly soluble drugs by forming inclusion complexes with their polar molecules or functional groups. The resulting complex hides most of the hydrophobic functionality in its interior cavity, while the hydrophilic hydroxyl groups get exposed to the environment [7]. Also, the bitter taste of substances can be reduced or even completely eliminated if they form inclusion complexes of sufficient stability with the selected cyclodextrin [8]. In the present study, this strategy of forming inclusion complexes of etoricoxib with BCD was investigated for taste masking as well as dissolution enhancement of the drug loaded in MDF. Although many studies have been reported on solubility enhancement and ODT of etoricoxib $[4,9]$ oral thin films of etoricoxib have not been formulated so far and hence our work is first of its kind.

\section{Materials and Methods}

2.1. Materials. Etoricoxib was the gift sample obtained from Glenmark Pharmaceuticals Ltd., Navi Mumbai, India. Betacyclodextrin (Kleptose) and sucralose were generously gifted by Par Pharmaceuticals, Chennai. Mixed fruit flavour 148691 and bitter masking flavour 689796 were kindly offered by Apex Laboratories, Chennai. HPLC grade methanol and acetonitrile were procured from Fisher Scientific. Distilled deionized water was used for formulation and analytical studies. All other reagents and chemicals used were of analytical reagent grade.

2.2. Drug-Excipient Compatibility Study. FTIR spectra of pure drugs, polymers used, and blends were recorded on KBr disk method using FTIR-8400S Spectrophotometer with IR solution software (Shimadzu, Japan) to confirm the compatibility between drug and excipients. Sample powder was thoroughly mixed by triturating with potassium bromide in a glass mortar with pestle and compressed into disks in a hydraulic press (Technosearch Instruments, India). FTIR spectra of all the samples were recorded over a spectral region from 4700 to $400 \mathrm{~cm}^{-1}$ using 20 scans with $4 \mathrm{~cm}^{-1}$ resolution.

2.3. Preparation of Inclusion Complex. Etoricoxib-beta-cyclodextrin inclusion complex at weight ratios ( $1 \mathrm{~g}: 1 \mathrm{~g})$ and molar ratio (1 molar: 1 molar) was prepared by kneading method. Kneaded products were obtained by triturating etoricoxib and beta-cyclodextrin in a glass mortar with the pestle by adding small volume of purified water. The slurry obtained was kneaded for $45 \mathrm{~min}$ and then dried in vacuum oven (Ketan Shivani Scientific Industries (P) Ltd., India) at $35^{\circ} \mathrm{C}$. Dried complex was passed through ASTM (American Standards for Testing of Materials) sieve number: 60 .

2.4. Formulation of Etoricoxib MDF. Mouth dissolving films of etoricoxib were prepared by solvent casting method using both the types of drug inclusion complexes of $\mathrm{BCD}$, the composition of which is given in Table 1 .
A homogenous solution of HPMC 15 cps was prepared by continuous stirring of the polymer solution in water. Sucralose, mixed fruit flavour, bitter masking flavour 148691, and glycerin were added to the above solution and stirred well. The solution was sonicated (Bath sonicator, Spinatech PVT Ltd, Italy) and kept aside for the removal of air bubbles. Accurately weighed etoricoxib-beta-cyclodextrin inclusion complex was added to the above solution and mixed well. By using pipette, $5 \mathrm{~mL}$ of prepared uniform dispersion was carefully transferred to the Petri dish $(8 \mathrm{~cm}$ diameter $)$ without air bubble and dried in microwave oven (Whirlpool, India) at $50^{\circ} \mathrm{C}$ for $45 \mathrm{~min}$. Further, the film was dried in vacuum oven (Ketan Shivani Scientific Industries (P) Ltd., India) at $35^{\circ} \mathrm{C}$. The prepared film was carefully removed from the Petri dish, checked for imperfections, and was cut into small circular films of $3 \mathrm{~cm}$ diameter that have an equivalent dose of $30 \mathrm{mg}$ etoricoxib per MDF. The samples were kept in butter paper and sealed with an Aluminium foil-aluminium foil pouch (alu-alu pouch).

2.5. Evaluation of Etoricoxib MDF. Etoricoxib MDFs were evaluated for uniformity of weight (Shimadzu Electronic Balance, Japan), thickness of film (Dial Gauge, model: K17, accuracy $0.001 \mathrm{~mm}$, Baker Precision Measuring Instruments, China), disintegration time (Digital Tablet Disintegration Test Apparatus, model: VTD-AV, Veego Instruments Corporation, India), In vitro dispersion time, surface $\mathrm{pH}$, folding endurance, water vapor permeability, and mechanical properties $[10,11]$.

2.5.1. Uniformity of Weight. Each film was individually weighed on analytical balance (Shimadzu Electronic Balance, Japan) and average weight of 3 films was found. A large difference in weight denotes the nonuniform distribution of drug in the film.

2.5.2. Thickness of Film. The thickness of the different films was measured using a calibrated dial gauge (Baker Precision Measuring Instruments, China) with an accuracy of 0.001 $\mathrm{mm}$. Thickness was measured by placing each film between the anvil and the presser foot of the dial gauge in 5 different locations and the average thickness was calculated.

2.5.3. In Vitro Dispersion Time. In vitro dispersion time was measured by Petri dish method. A film was dropped in culture dish of $8 \mathrm{~cm}$ in diameter, containing $10 \mathrm{~mL}$ of simulated salivary fluid. The mean in vitro dispersion time of 6 films was determined [12].

2.5.4. Surface $p H$. Either highly acidic or highly basic $\mathrm{pH}$ of MDF would cause discomfort on administration. To know the surface $\mathrm{pH}$ of the film, the film was placed in a Petri dish and was moistened with $0.5 \mathrm{~mL}$ of distilled water and kept for $30 \mathrm{sec}$. The surface $\mathrm{pH}$ was measured by means of $\mathrm{pH}$ paper placed on the surface of the swollen films. The average of 3 determinations for each formulation was found out. 
TABLE 1: Formulation of etoricoxib MDF.

\begin{tabular}{|c|c|c|c|}
\hline \multicolumn{2}{|r|}{ Batch code } & EF-1 & EF-2 \\
\hline S. number & Ingredients & \multicolumn{2}{|c|}{ Quantity/film } \\
\hline 1. & Etoricoxib-beta-cyclodextrin complex & $426.7 \mathrm{mg}^{*}$ & $886.4 \mathrm{mg}^{*}$ \\
\hline 2. & Hypromellose (hydroxypropyl methyl cellulose $15 \mathrm{cPs}$ ) & $150 \mathrm{mg}$ & $150 \mathrm{mg}$ \\
\hline 3. & Sucralose & $50 \mathrm{mg}$ & $50 \mathrm{mg}$ \\
\hline 4. & Mixed fruit flavour 148691 & $0.025 \mathrm{~mL}$ & $0.025 \mathrm{~mL}$ \\
\hline 5. & Bitter masking flavour 689796 & $0.025 \mathrm{~mL}$ & $0.025 \mathrm{~mL}$ \\
\hline 6. & Glycerin & $65 \mathrm{mg}$ & $65 \mathrm{mg}$ \\
\hline 7. & Distilled water & $5 \mathrm{~mL}$ & $5 \mathrm{~mL}$ \\
\hline
\end{tabular}

${ }^{*}$ Quantity equivalent to $30 \mathrm{mg}$ of etoricoxib in single dose MDF. EF-1-MDF with beta-cyclodextrin inclusion complex (1:1g ratio). EF-2-MDF with betacyclodextrin inclusion complex (1:1 molar ratio).

2.5.5. Folding Endurance. Folding endurance was measured manually for the prepared films. A $7.07 \mathrm{~cm}^{2} \mathrm{MDF}$ was repeatedly folded at $180^{\circ} \mathrm{C}$ angle of the plane at the same place until it breaks. The number of times the film could be folded at the same place without breaking was noted for 3 films of same batch [13].

2.5.6. Water Vapor Permeability. Water vapor transmission rate (WVTR) of the film was measured by modified ASTM E96 method. The film was sealed on the top of a glass vial (4 mL) containing $2.5 \mathrm{~mL}$ of distilled water $(100 \% \mathrm{RH} ; 3169 \mathrm{~Pa}$ vapor pressure at $25^{\circ} \mathrm{C}$ ), which was placed in a desiccator at $25^{\circ} \mathrm{C}$ and $0 \% \mathrm{RH}$ containing fused calcium chloride $(0 \mathrm{~Pa}$ water vapor pressure). The vials are weighed every $24 \mathrm{hrs}$ for 1 week. The amount of water vapor permeated through the films was determined from the weight loss [14, 15]. WVTR and water vapor permeability (WVP) were calculated using the formula

$$
\begin{aligned}
& \mathrm{WVTR}=\frac{\Delta w}{\Delta t} \times A \\
& \mathrm{WVP}=\frac{\mathrm{WVTR} \cdot L}{\Delta p},
\end{aligned}
$$

where WVTR is in $\mathrm{g} / \mathrm{h} \mathrm{m}^{2}, \Delta w / \Delta t$ is rate of water gain in $\mathrm{g} / \mathrm{h}$, $A$ is the exposed area of the film in $\mathrm{m}^{2}, L$ is the mean thickness of film specimens in $\mathrm{m}$, and $\Delta p$ is the difference in partial water vapor pressure between the two sides of film specimens in $\mathrm{Pa}$. The water vapor pressure on the high-stream side of the film was $3.169 \mathrm{kPa}$ (i.e., saturated water vapor pressure at $25^{\circ} \mathrm{C}$ ), while the low-stream side is assumed to be zero. Three replicates of the determinations were done.

2.5.7. Mechanical Properties of Films. Mechanical properties of films were evaluated by Texture Analyzer (TAXT plus, Stable Microsystems, UK; maximum load $50 \mathrm{~kg}$ ).

(a) Burst Strength. Film burst strength is the force required to break or rupture the film, which is an indicator of the flexibility of the film. Burst strength of the film was studied using $5 \mathrm{~mm}$ spherical stainless steel ball probe $(\mathrm{P} / 5 \mathrm{~S})$ with the probe adapter which was connected to the load cell [16]. A circular stripfilm with an area of $7.07 \mathrm{~cm}^{2}$ was placed in film supporting rig and moving probe reached the surface of the film with the pretest speed of $2.0 \mathrm{~mm} / \mathrm{sec}$. When the probe reached the surface of the film, probe speed was changed to $1.0 \mathrm{~mm} / \mathrm{sec}$ test speed with the trigger load of $5 \mathrm{~g}$ and the data were recorded.

(b) Tensile Strength. Tensile strength of a film is an indicator of toughness of film [17]. Etoricoxib films were cut into specimens with a width of $20 \mathrm{~mm}$ and a length of $60 \mathrm{~mm}$. Film thickness was measured by means of calibrated dial gauge with an accuracy of $0.001 \mathrm{~mm}$ at 5 different positions. Tensile strength of the film was determined with Tensile Grips (A/TG). The test film was fixed to the upper tensile grip and load cell was tared to zero weight. Upper tensile grip was moved to the preset distance of $25 \mathrm{~mm}$ and the test film was securely clamped to lower grip. The tensile force was gradually applied on the test film till the film broke. The parameters maintained were $1 \mathrm{~mm} / \mathrm{s}$ pretest speed, $1 \mathrm{~mm} / \mathrm{s}$ test speed, in distance target mode [18].

Tensile strength was calculated using the formula

$$
\text { Tensile Strength }=\frac{F_{\max }}{A_{\text {film }}},
$$

where " $F_{\max }$ " is the maximum force at breakage $(N)$ and " $A_{\text {film }}$ " is the initial cross sectional area of the sample $\left(\mathrm{mm}^{2}\right)$. The data reported is the mean of 3 individual determinations.

$\%$ elongation was calculated using the formula

$$
\text { \% Elongation }=\frac{L-L_{0}}{L_{0}} \times 100,
$$

where " $L_{0}$ " is the initial gauge length of the specimen (mm) and " $L$ " is the length at the moment of rupture $(\mathrm{mm})$.

Young's modulus was calculated using the formula

$$
\text { Young's modulus }=\left(\frac{F_{\text {lin }}}{A_{\text {film }}}\right) \times\left(\frac{1}{\epsilon}\right),
$$

where " $F_{\text {lin }}$ " is the force at corresponding strain of the linear section $(\mathrm{N})$, " $A_{\text {film }}$ " is the initial cross sectional area of the sample $\left(\mathrm{mm}^{2}\right)$, and $\in$ is the corresponding strain.

2.6. HPLC Analysis. A HPLC method was used in determination of drug content of films and analysis of samples in 
TABLE 2: Taste evaluation reference table.

\begin{tabular}{|c|c|c|c|c|c|}
\hline \multirow{2}{*}{ Parameters } & \multicolumn{5}{|c|}{ Score } \\
\hline & 1 & 2 & 3 & 4 & 5 \\
\hline Bitterness $^{*}$ & Extremely bitter & Highly Bitter & Acceptable & Very slightly bitter & Not at all bitter \\
\hline Sweetness ${ }^{*}$ & Not at all sweet & Very slightly sweet & Acceptable & Highly sweet & Extremely sweet \\
\hline Mouth feel & Very gritty & Gritty & Acceptable & Creamy & Very creamy \\
\hline Flavor & Very unpleasant & Unpleasant & Acceptable & Pleasant & Very pleasant \\
\hline Overall acceptability & Worst & Poor & Acceptable & Good & Very good \\
\hline
\end{tabular}

* Parameter assessed for initial taste and after bitterness.

drug release studies using HPLC (Waters 2695 series, USA, PDA Detector (Waters 2996 PDA, USA)) and an integrator (Empower). The mobile phase was a mixture of two in the proportion $60: 40$ : the first one was the buffer of $\mathrm{pH} 2.5$ prepared with $0.1 \%$ Di basic sodium phosphate, $0.1 \%$ ammonium acetate, and $0.1 \%$ sodium pentanesulfonate filtered through 0.45 micron filter and the second one was acetonitrile and methanol mixed in the proportion of $20: 20$, sonicated, and degassed for 10 minutes by using sonicator. The stationary phase was waters symmetry shield, C-18 column, $250 \times$ $4.6 \mathrm{~mm}, 5 \mu \mathrm{m}$, Waters, USA. The injection volume was $50 \mu \mathrm{L}$ and the flow rate was $1 \mathrm{~mL} / \mathrm{min}$. The column was maintained at $25^{\circ} \mathrm{C}$ and the effluent was monitored at $234 \mathrm{~nm}[19,20]$.

2.7. Drug Content and Uniformity of Dosage Units. A film was taken in a $100 \mathrm{~mL}$ volumetric flask and sonicated with $70 \mathrm{~mL}$ of methanol for 5 minutes after which the volume was made up to $100 \mathrm{~mL}$ with methanol. Then $1.0 \mathrm{~mL}$ of this solution was diluted to $100 \mathrm{~mL}$ with $0.1 \mathrm{~N}$ hydrochloric acid which was filtered through 0.45 micron filter and diluted as required and the drug content was found out by HPLC analysis.

2.8. In Vitro Drug Dissolution Study. The dissolution studies were performed in $900 \mathrm{~mL}$ of simulated salivary fluid as well as $0.1 \mathrm{~N}$ hydrochloric acid using Lab India DS8000 dissolution (paddle) apparatus (Lab India Instruments Pvt. Ltd., India) with autosampler at $37 \pm 0.5^{\circ} \mathrm{C}$ with paddle rotation speed at $50 \mathrm{rpm}$. The samples were collected through built-in $10 \mu$ filter which were diluted previous to HPLC analysis.

\subsection{Evaluation of Taste Masking and In Vivo Disintegration} Time. Taste masking was assessed by ten human volunteers from whom informed consent was obtained and they had participated in the test under the supervision of a clinician and the study protocol was approved by Institutional Ethical Committee, Ultra College of Pharmacy, Madurai (UCP/IEC/ 2013-2014/29). Volunteers were asked to rinse their mouth with a cup of water $(200 \mathrm{~mL})$ before the test and instructed to move the dose against the upper part of the mouth with the tongue without biting. They were also instructed to spit the contents, when the dose got disintegrated [21]. Volunteers were asked to rate the initial taste, after taste, mouth feel, flavour, and overall acceptability of formulations as per the ratings given in Table 2 .
2.10. Differential Scanning Calorimetry (DSC). The powdered sample (2-3 mg) of etoricoxib, BCD, inclusion complex, and dry mix of etoricoxib MDF were hermetically sealed in aluminum pans and heated at a constant rate of $5^{\circ} \mathrm{C} / \mathrm{min}$, over a temperature range of $0-300^{\circ} \mathrm{C}$. Thermogram of the samples was obtained using differential scanning calorimetry (DSC, Q20, TA Instruments, USA). Thermal analysis data were recorded using Universal Software. Indium standard was used to calibrate the DSC temperature and enthalpy scale. Aluminum pan with lid was used for all samples. An empty aluminum pan was used as reference.

2.11. Powder X-Ray Diffraction (PXRD). The powder XRD patterns of etoricoxib, BCD, inclusion complex, dry mix of etoricoxib MDF, and etoricoxib MDF were obtained from an X-ray diffractometer (Rigaku MiniFlex 600, Japan) working with $\mathrm{Cu}-\mathrm{K} \alpha$ radiation and in $2 \theta$ range of $10-90^{\circ}$ at $40 \mathrm{kV}$ and $15 \mathrm{~mA}$. The scan duration time was $10 \mathrm{Deg} . / \mathrm{min}$ with step size of 0.020 . The diffracted radiation from the samples passed through $1.25^{\circ}$ divergence slit and $0.30 \mathrm{~mm}$ receiving slit.

2.12. Scanning Electron Microscopy (SEM). SEM studies on etoricoxib, BCD, drug-BCD inclusion complex, and etoricoxib MDF were done with the Scanning Electron Microscope (Tescan, Vega 3 SBH, Czech Republic). The samples were mounted onto aluminum stubs using carbon doublesided tape, gold coated with a sputter coater (Quorum sputter coater, SC7620, UK), and examined at an excitation voltage of $5 \mathrm{kV}$.

2.13. Evaluation of Analgesic Activity of Etoricoxib MDF in Wistar Albino Rats. The analgesic activity of the test solutions of etoricoxib formulations was evaluated by tail flick test in Wistar albino rats. The experimental procedures and protocols followed in the animal studies were reviewed and approved (Proposal number UCP/IAEC/2012/064) by the Institutional Animal Ethical Committee (IAEC) of Ultra College of Pharmacy, Madurai, constituted in accordance with the guidelines of the CPCSEA, Government of India Registration number 890/ac/05/CPCSEA.

2.13.1. Experimental Animals. Healthy Wistar albino rats of either sex weighing 150-200 g were used for the determination of analgesic activity of etoricoxib MDF. The animals were housed comfortably in a group of five in a single clean polypropylene cage (internal dimensions $33.6 \mathrm{~cm} \times 23.2 \mathrm{~cm}$ 
$\times 15.4 \mathrm{~cm}, L \times W \times H)$ with a metal frame lid on its top. They were housed in an environmentally controlled room (temperature $24 \pm 1^{\circ} \mathrm{C}$; relative humidity $30-70 \%$.) under a 12-hour light/dark cycle. The animals were fed on standard pelletized laboratory animal diet and tap water ad libitum. The animals were used after an acclimatization period of 7 days to the laboratory environment. Each rat was conditioned for $30 \mathrm{~min}$ in the restrainer before starting the experiment [22].

2.13.2. Tail Flick Test. The ventral surface of the tail of the animal (approximately $5 \mathrm{~cm}$ from the caudal end of the tail) was placed on the heating coil $\left(45^{\circ} \pm 2^{\circ} \mathrm{C}\right)$ of digital Analgesiometer (Instruments and Chemicals Pvt. Ltd., India) and the basal reaction times were noted. About 3-5 basal reaction times were noted for each rat at a gap of $5 \mathrm{~min}$ to confirm the normal behavior of the animal [23].

Based on previously available literature, the dose of etoricoxib was fixed as $10 \mathrm{mg} / \mathrm{kg}$ body weight [24]. Etoricoxib film and commercial tablets were dissolved in purified water and calculated equivalent dose was given to albino rats orally, using syringe and feeding needle. Tail flicking response of albino rats was observed for every 10 minutes for 1 hour. As the reaction time reaches $10 \mathrm{sec}$, it was considered as maximum analgesia and the tail was removed from the source of heat to avoid tissue damage. Analgesic activity was quantified by finding out the relative increase in reaction time to the maximum response ( $10 \mathrm{sec}$, reaction time) which was calculated at each time interval using the formula given below:

$$
\text { Index of analgesia in } \%=\left[\frac{(\text { Reaction time at a particular time interval of testing }- \text { Basal reaction time })}{(10-\text { Basal reaction time })}\right] \times 100 \text {. }
$$

\section{Results and Discussion}

3.1. Drug-Excipient Compatibility Study. IR spectra of etoricoxib, BCD, inclusion complex, and dry mix of MDF are given in Figure 1. The FTIR spectrum of pure etoricoxib drug showed characteristic peaks of aromatic $\mathrm{C}=\mathrm{C}$ stretching at $1597.11 \mathrm{~cm}^{-1}, 1562.39 \mathrm{~cm}^{-1}, 1539.25 \mathrm{~cm}^{-1}$, and $1492.95 \mathrm{~cm}^{-1}$. Prominent peaks at $1145.75 \mathrm{~cm}^{-1}, 1084.03 \mathrm{~cm}^{-1}$ indicate $\mathrm{S}=\mathrm{O}$ stretching vibrations. $\mathrm{C}-\mathrm{Cl}$ stretching vibration was denoted by the presence of peak at $775.41 \mathrm{~cm}^{-1}$. Absorption peak of C-H stretching vibration was observed at $2962.76 \mathrm{~cm}^{-1}$ and $\left(\mathrm{CH}_{3}\right) \mathrm{C}-\mathrm{H}$ stretching vibrations were observed at $1427.37 \mathrm{~cm}^{-1}$. Also $\mathrm{C}-\mathrm{H}$ deformation peak was found at $850.64 \mathrm{~cm}^{-1}$. The obtained FTIR spectrum thus confirms the purity of the drug.

The FTIR spectrum of beta-cyclodextrin shows prominent peaks of $\mathrm{O}-\mathrm{H}$ stretching vibration at $3375.54 \mathrm{~cm}^{-1}$. C$\mathrm{H}$ stretching and $\mathrm{C}-\mathrm{O}$ stretching vibrations were observed at $2962.11 \mathrm{~cm}^{-1}$ and $1647.26 \mathrm{~cm}^{-1}$, respectively. Absorption peaks of $\mathrm{C}-\mathrm{O}-\mathrm{C}$ stretching vibrations were located at $1249.91 \mathrm{~cm}^{-1}$ and $1157.33 \mathrm{~cm}^{-1}$.

The IR spectrum of etoricoxib-beta-cyclodextrin complex bears the peaks corresponding to the etoricoxib peaks as well as that of beta-cyclodextrin with no significant shift in the major peaks. The FTIR spectrum of dry mix of etoricoxib MDF shows all the prominent peaks of etoricoxib indicating the maintenance of identity of the drug and thus the stability of the drug in film [25].

3.2. Preparation of Etoricoxib MDF. The drug-BCD complexes prepared in molar ratio and weight ratio of $1: 1$ were mixed with $3 \% \mathrm{w} / \mathrm{v}$ solution of HPMC $15 \mathrm{cPs}$ solution separately to obtain two formulations. The films were prepared by solvent casting method. The removal of etoricoxib MDF with 1:1 molar ratio of beta-cyclodextrin inclusion complex from the glass substrate (Petri dish) was very difficult due to the presence of high solids content. So etoricoxib MDF formulated with $1: 1$ weight ratio $\mathrm{BCD}$ complex was used for further studies.

3.3. Evaluation of Etoricoxib MDF. The physicomechanical properties of etoricoxib MDF prepared with $1: 1 \mathrm{~g} / \mathrm{g}$ drug: beta-cyclodextrin (EF-1) are presented in Table 3.

3.3.1. Appearance, Weight, and Thickness. The prepared films were homogenous, colourless, smooth, and rough surface. The weight variation was found to be minimum as indicated by a small standard deviation of $\pm 1.66 \mathrm{mg}$. This observation also shows the uniform distribution of the ingredients in MDF. The thickness shows a narrow range of 120.92 to $128.28 \mu \mathrm{m}$ further substantiating the above inference.

3.3.2. Mechanical Properties of Film. Mouth dissolving films must be strong enough and ductile to prevent the rupture of the dosage form during processing and packing and during transit. The good burst strength and young modulus values of etoricoxib MDF results indicate that etoricoxib films are flexible and at the same time rigid enough to prevent the deformation of the dosage form.

Tensile strength, \% elongation, and folding endurance results indicate that etoricoxib MDF is slightly elastic and breaks at low force which might be due to the presence of high solid content [17].

Surface $\mathrm{pH}$ of the film is the neutral $\mathrm{pH}$ so that the films are safe to be used in buccal cavity without any problem of irritation and thus patient acceptance will not be affected. Water vapour permeability is a measure of ease of the moisture to pass through a material. The high water vapour permeability of etoricoxib MDF would have been due to the presence of pores in MDF [14] which is also confirmed by SEM images as discussed in another following section. 


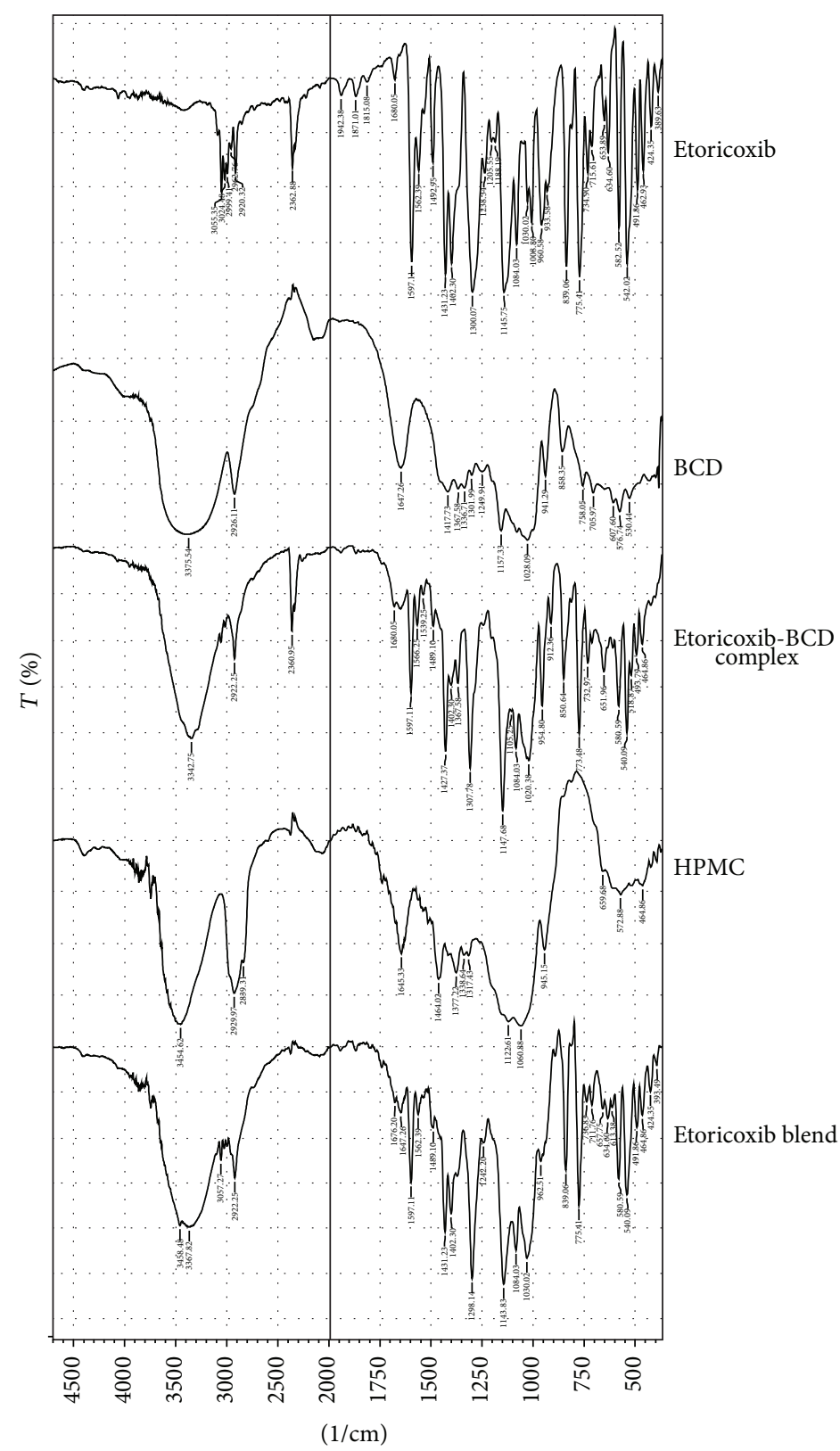

FIGURE 1: IR spectra of etoricoxib, BCD, etoricoxib-BCD complex, HPMC and dry mix of etoricoxib MDF.

3.4. In Vitro Dispersion Time and Disintegration Time. In vitro dispersion time of etoricoxib MDF ranges from 31 to $38 \mathrm{sec}$. The FDA recommends a disintegration time of $30 \mathrm{~s}$ or less for ODTs based on the USP disintegration test [26]. Etoricoxib MDF showed disintegration time of $8-9 \mathrm{sec}$ and passes the limit for disintegration time.

3.5. Drug Content and UOD. Drug content of etoricoxib MDF was in the range of $99.0-100.6 \%$ implying uniform distribution of drug in the films.

3.6. In Vitro Drug Release Study. In vitro drug release studies of etoricoxib MDF were carried out in simulated salivary fluid
(SSF) and $0.1 \mathrm{~N}$ hydrochloric acid for 30 minutes. Etoricoxib MDF showed rapid and complete release profiles in both the media which correlates well with both disintegration time and in vitro dispersion time. Dissolution profiles of etoricoxib from MDF in SSF and $0.1 \mathrm{~N} \mathrm{HCl}$ are given in Figures 2(a) and 2(b), respectively.

3.7. Taste Evaluation and In Vivo Disintegration Time. Results of in vivo disintegration time and palatability evaluation are presented in Table 4.

Results of in vivo disintegration time indicate that etoricoxib MDF disintegrated within 30 seconds and correlated well with in vitro dispersion time and disintegration time. In vivo disintegration time of etoricoxib MDF was 7.2-8.4 sec 


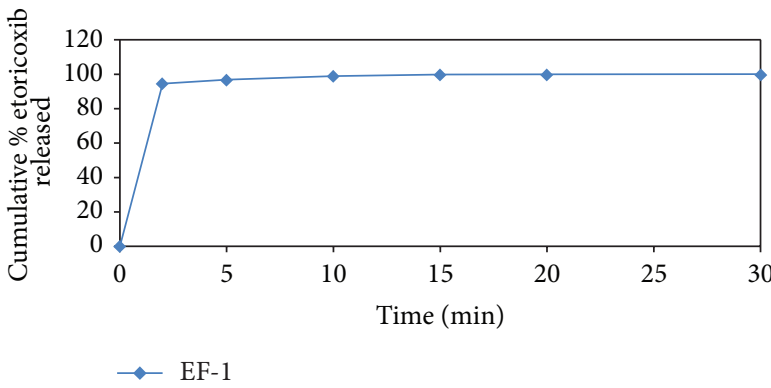

(a)

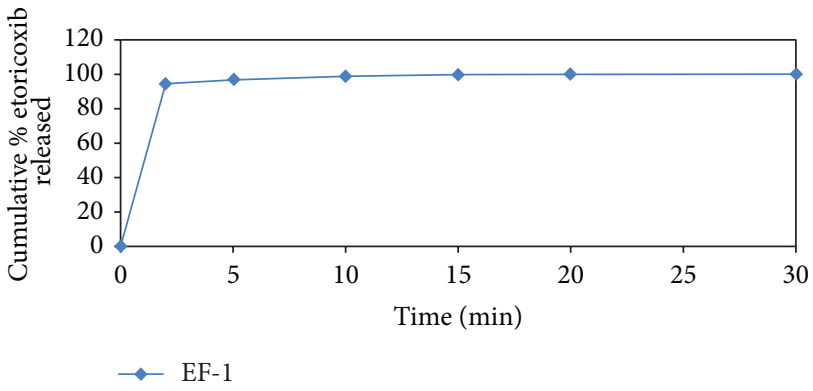

(b)

FIGURE 2: In vitro drug release of etoricoxib MDF (EF-1) in (a) simulated salivary fluid and (b) $0.1 \mathrm{~N} \mathrm{HCl}$.

TABLE 3: Physical and mechanical characterization of etoricoxib MDF (EF-1).

\begin{tabular}{lc}
\hline Parameters & \\
\hline Weight $(\mathrm{mg})$ & $93.4 \pm 1.66$ \\
Thickness $(\mu \mathrm{m})$ & $124.6 \pm 3.68$ \\
Burst strength $(\mathrm{N})$ & $0.09 \pm 0.001$ \\
Tensile strength $(\mathrm{MPa})$ & $4.0 \pm 1.58$ \\
$\%$ elongation & $0.7 \pm 0.24$ \\
Young's modulus $(\mathrm{MPa})$ & $375.8 \pm 3.03$ \\
Folding endurance & $2 \pm 1$ \\
Surface pH & 7 \\
Water vapor permeability $\left(\mathrm{g} \cdot \mathrm{m} / \mathrm{Pa} \mathrm{h} \mathrm{m}{ }^{2}\right) \times 10^{-7}$ & 17.8 \\
In vitro dispersion time $(\mathrm{sec})$ & $34.6 \pm 3.70$ \\
Disintegration time $(\mathrm{sec})$ & $8.5 \pm 0.70$ \\
\hline
\end{tabular}

${ }^{*}$ Values represent the mean $\pm \mathrm{SD}$

which is even lower than the in vitro disintegration time suggesting the aided factor to be the pressing movement of tongue with the palate while keeping the film in position.

The results of taste evaluation study indicate that the taste masking of etoricoxib could be achieved with complexation with beta-cyclodextrin. Although the dissolution of etoricoxib MDF in SSF was rapid, the bitterness was not perceived by the volunteers as shown by the results. This is due to the fact that etoricoxib trapped in the inclusion complex formed with BCD was not exposed to taste buds during dispersion. The flavours also must have contributed to effective taste masking. The immediate dispersion and dissolution of MDF may produce rapid therapeutic action through faster drug absorption right from the buccal cavity.

Further, to characterize the inclusion complex and film, DSC and PXRD studies were carried out on drug, BCD, inclusion complex, film blend, and films of etoricoxib MDF.

3.8. Differential Scanning Calorimetry (DSC). DSC of etoricoxib MDF is presented in Figure 3. Melting of etoricoxib in DSC starts at $137^{\circ} \mathrm{C}$. DSC curve of etoricoxib exhibited a broad endothermic peak at $147.15^{\circ} \mathrm{C}$, starting at which is ascribed to drug melting [27]. DSC curve of beta-cyclodextrin exhibited a broad endothermic peak at $160^{\circ} \mathrm{C}$, which is due to its dehydration of bound water.
DSC thermogram of etoricoxib and beta-cyclodextrin complex showed characteristic changes in melting endotherm and its enthalpy. Both the endothermic peaks were broadened and shifted to lower temperature with reduced intensity and enthalpy. This could be due to the possible interaction between etoricoxib and beta-cyclodextrin and loss of crystallinity. Etoricoxib endotherm was shifted from $147.15^{\circ} \mathrm{C}$ to $118.61^{\circ} \mathrm{C}$ and beta-cyclodextrin endothermic peak shifted from $160^{\circ} \mathrm{C}$ to $157.63^{\circ} \mathrm{C}$. Enthalpy reductions observed were from $47.00 \mathrm{~J} / \mathrm{g}$ to $32.15 \mathrm{~J} / \mathrm{g}$ and from $872.3 \mathrm{~J} / \mathrm{g}$ to $33.03 \mathrm{~J} / \mathrm{g}$ for etoricoxib and beta-cyclodextrin, respectively.

Dry mix of etoricoxib-MDF blend retained the peak of beta-cyclodextrin at $157^{\circ} \mathrm{C}$, whereas the merged/broad peak starts melting from $126.59^{\circ} \mathrm{C}$ and appeared as a peak at $141.71^{\circ} \mathrm{C}$ which might be due to etoricoxib in BCD complex and HPMC.

DSC thermogram of etoricoxib MDF showed endothermic peak of $135^{\circ} \mathrm{C}$ and $110.43^{\circ} \mathrm{C}$ which might be due to betacyclodextrin and etoricoxib in BCD complex and HPMC, respectively. Both the endothermic peaks were broadened and shifted to lower temperature with reduced intensity. This could be due to the possible interaction between HPMC and etoricoxib-BCD complex and further loss of crystallinity. A peak at $91^{\circ} \mathrm{C}$ indicates the loss of moisture in the sample.

3.9. Powder X-Ray Diffraction. Powder X-ray diffractograms (PXRD) of etoricoxib, BCD, etoricoxib-beta-cyclodextrin complex, HPMC, etoricoxib-MDF dry mix, and etoricoxib MDF are given in Figure 4. PXRD of etoricoxib reveals many distinct reflections pointing to its highly crystalline nature. Various diffraction peaks of the drug crystals can be traced in the spectrum of the pure drug at $2 \theta$ values of $12.2,12.8$, $13.4,15.9,16.9,18.5,19.7,20.4,21.5,23.1,24.0,24.4,26.7,28.7$, 29.6, 30.2, 31.1, 31.6, 32.3, 36.1, 39.6, 43.4, 45.5, and 47.2. The crystalline nature of BCD is also revealed by the PXRD of beta-cyclodextrin as shown by many distinct reflections. Various diffraction peaks of the drug crystals can be traced in the spectrum of the pure drug at $2 \theta$ values of $10.9,11.9,12.9$, $13.9,15.0,15.7,16.4,17.1,17.4,17.9,1.4,19.2,19.9,21.5,23.0,24.6$, $25.3,25.9,27.4,28.8,30.6,31.3,32.2,35.1,36.2,38.0,39.7,40.8$, $42.8,44.6,45.3$, and 51.5. 
TABLE 4: Taste evaluation by human volunteers.

\begin{tabular}{|c|c|c|c|c|}
\hline \multirow{3}{*}{ In vivo disintegration time ( $\mathrm{sec}$ ) } & \multicolumn{4}{|c|}{ Average score given by the human volunteers } \\
\hline & \multicolumn{4}{|c|}{ Initial observation } \\
\hline & Bitterness & Sweetness & Mouth feel & Flavour \\
\hline \multirow{4}{*}{$7.8 \pm 0.63$} & $4.6 \pm 0.52$ & $4.6 \pm 0.52$ & $4.7 \pm 0.48$ & $4.8 \pm 0.42$ \\
\hline & \multicolumn{4}{|c|}{ After 5 minutes } \\
\hline & Bitterness & Sweetness & \multicolumn{2}{|c|}{ Overall acceptability } \\
\hline & $4.1 \pm 0.30$ & $4.6 \pm 0.49$ & \multicolumn{2}{|c|}{$4.7 \pm 0.46$} \\
\hline
\end{tabular}

Values are given as mean $\pm \operatorname{SEM}(n=10)$.

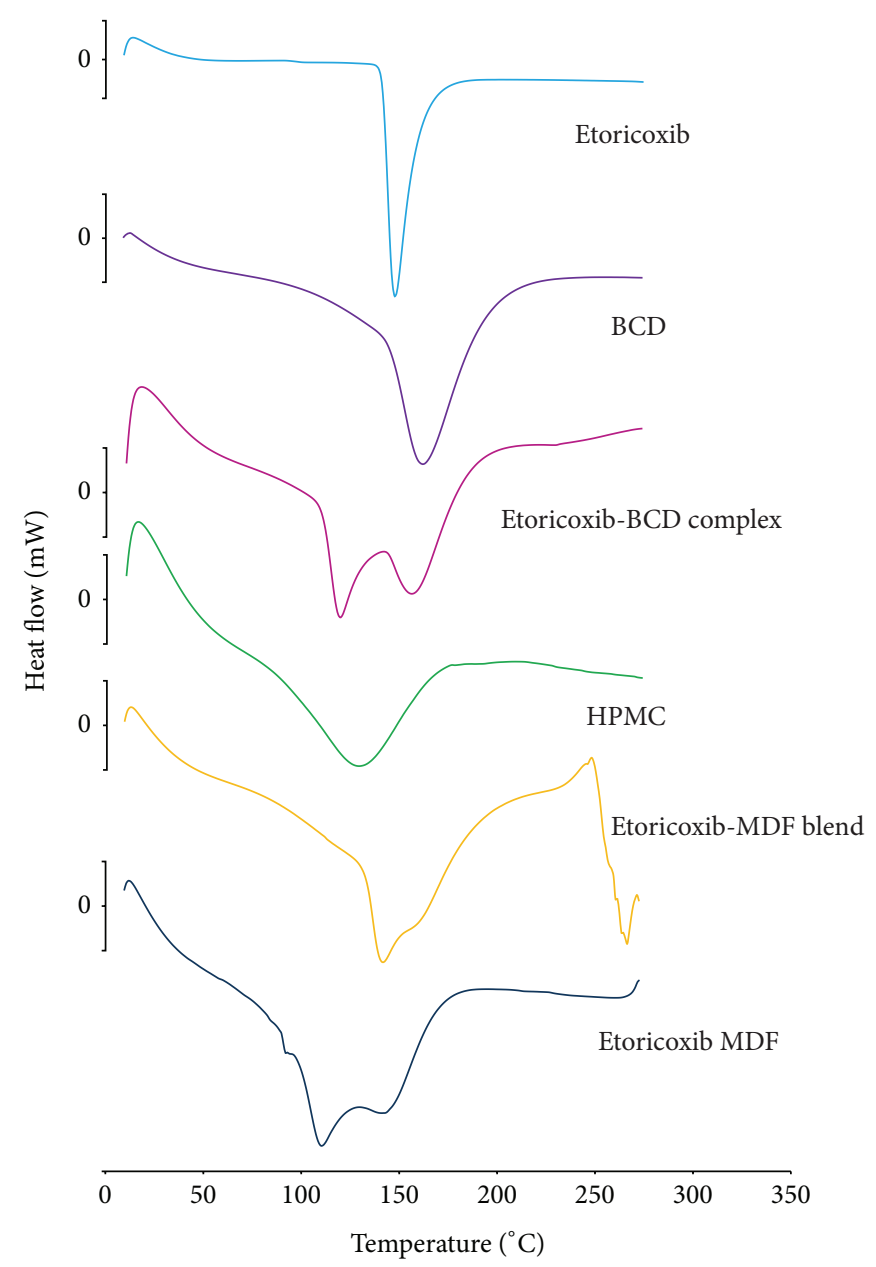

FIgURE 3: Differential scanning calorimetry profiles of etoricoxib MDF.

Prominent peaks at $2 \theta$ values of $18.5,12.2,15.7$, and 24.4 are missing in the diffractogram of etoricoxib-betacyclodextrin complex. Intensity of peaks and peak heights are reduced at 16.9, 12.8, and 23.1. Reductions in peak intensity, shifts and absence of peaks, presence of new diffraction peaks, or a complete diffuse pattern might be related to possible change of crystallinity of the drug to amorphous form and/or complexation. An increase in peak width stands for the reduction in particle size $[28,29]$.

In PXRD of etoricoxib film blend, new peaks are present along with that of drug which might be due to the crystalline excipients in blend composition. Absence of peaks, decrease in peak intensity, and peak height of pure drug indicate reduction in drug crystallinity.

PXRD of etoricoxib film shows drug peaks at $2 \theta$ values of 16.9 and 24.0 with decreased intensity. All the other peaks corresponding to the drug were absent in the diffractogram suggesting the loss of crystallinity of the drug.

3.10. Scanning Electron Microscopy (SEM). SEM images of etoricoxib, BCD, drug-BCD inclusion complex, and etoricoxib MDF are presented in Figure 5. SEM of etoricoxib exposes discrete, elongated flake-like structures with rough 
TABLE 5: Evaluation of analgesic activity by tail flick test (reaction time).

\begin{tabular}{lcccccc}
\hline \multirow{2}{*}{ Treatment } & \multirow{2}{*}{ Basal reaction time $(\mathrm{Sec})$} & \multicolumn{4}{c}{ Reaction time (sec) } \\
& & $10 \mathrm{~min}$ & $20 \mathrm{~min}$ & $30 \mathrm{~min}$ & $40 \mathrm{~min}$ & $50 \mathrm{~min}$ \\
\hline Control & $1.26 \pm 0.06$ & $1.27 \pm 0.11$ & $1.22 \pm 0.05$ & $1.25 \pm 0.09$ & $1.27 \pm 0.08$ & $1.19 \pm 0.04$ \\
Marketed IR tablet & $1.44 \pm 0.31$ & $1.44 \pm 0.25^{\mathrm{ns}}$ & $3.16 \pm 0.36^{\mathrm{a}}(20 \%)$ & $8.83 \pm 0.35^{\mathrm{b}}(86 \%)$ & $10.17 \pm 0.04^{\mathrm{b}}(102 \%)$ & $>10 \mathrm{sec}(>100 \%)$ \\
Etoricoxib MDF & $1.26 \pm 0.14$ & $1.58 \pm 0.28^{\mathrm{ns}}$ & $3.53 \pm 0.26^{\mathrm{a}}(26 \%)$ & $8.85 \pm 0.50^{\mathrm{b}}(87 \%)$ & $10.18 \pm 0.16^{\mathrm{b}}(102 \%)$ & $>10 \mathrm{sec}(>100 \%)$ \\
\hline
\end{tabular}

Values are expressed as mean $\pm \mathrm{SD}(n=3) ;{ }^{\mathrm{a}} \mathrm{P}<0.01$; ${ }^{\mathrm{b}} \mathrm{P}<0.001$; ns: not significant when compared to control; data were analyzed by using one-way ANOVA followed by Tukey-Kramer multiple comparison test.

The values in the parentheses indicate the percentage increase in reaction time.

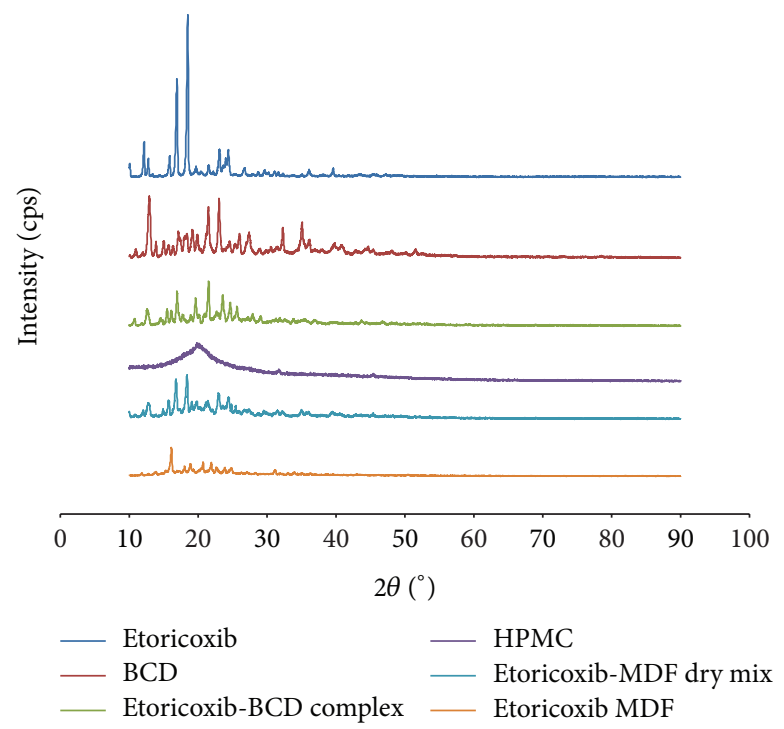

FIGURE 4: X-ray diffraction pattern of etoricoxib, BCD, etoricoxib-BCD complex, HPMC, etoricoxib-MDF dry mix, and etoricoxib MDF.

edges covered on their surfaces by fine particles. Some structures are large with parallelogram shape. It also reveals the hard and thick nature of the drug particles. In contrast, etoricoxib-BCD complex observed by SEM is soft and thin. Agglomerates of particles clumping to each other are present. The forms are different from both of the drug and BCD with reduced particles size. SEM of etoricoxib MDF shows the rough and uneven surface with circular pits with the absence of particles suggesting the presence of the drug in dissolved state in the polymer HPMC.

The results of DSC, XRD, and SEM confirm the reduction in particle size of etoricoxib in inclusion complex. They further ensure the loss of crystallinity when formulated as a film comprising amorphous HPMC.

3.11. Evaluation of Analgesic Activity of Etoricoxib MDF in Animal Studies. Analgesic activity of etoricoxib MDF was studied by tail flick test in Wistar rats. Results of tail flick test are presented in Table 5 . Results were statistically analyzed by one-way ANOVA, followed by the Tukey-Kramer multiple comparison test using GraphPad InStat software. $P<0.001$ was considered extremely significant.
The statistical data showed that there is no significant analgesic activity for both etoricoxib MDF and commercially available immediate release tablets at 10 minutes when compared to control $(P>0.05)$. But both formulations showed significant activity at $20 \mathrm{~min}$ when compared to the control $(P<0.01)$. The results demonstrate the presence of drug in the dissolved state leading to immediate absorption followed by fast analgesia greater than/comparable to that of IR tablets of etoricoxib. Thus, better management of pain will be possible with the developed etoricoxib MDF.

\section{Conclusion}

To conclude, the developed etoricoxib MDF with both enhanced dissolution and acceptable taste masking was achieved by forming inclusion complex with beta-cyclodextrin in $1: 1 \mathrm{~g}$ ratio. The cause for the improved dissolution of the drug has been shown to be particle size reduction of the drug in inclusion complex as shown by physical characterizations. DSC, PXRD, and SEM studies also confirm the formation of inclusion complex and change in crystallinity. Etoricoxib MDF prepared possesses adequate mechanical 


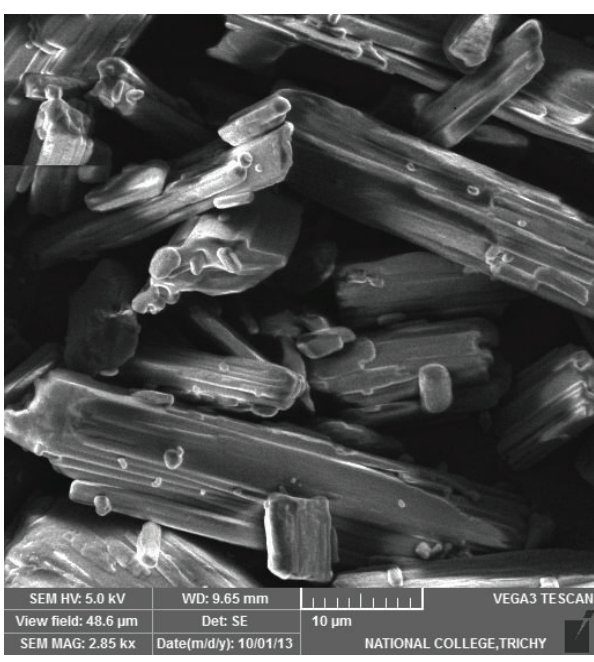

(a)

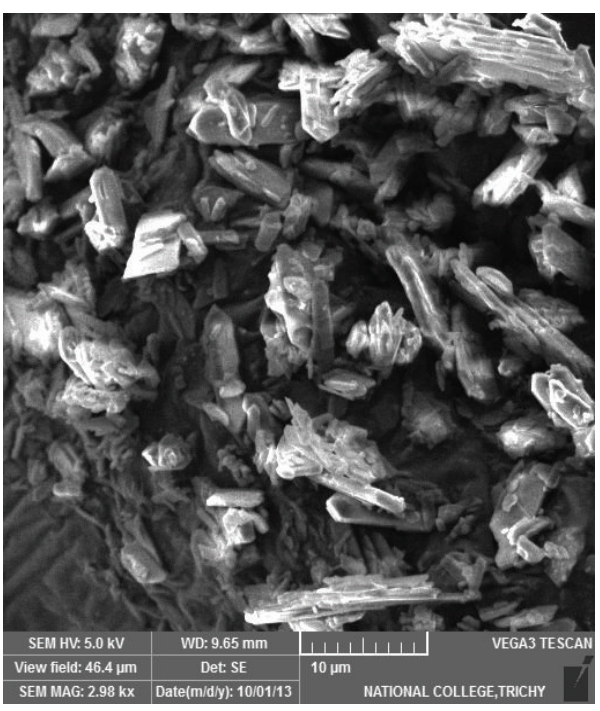

(c)

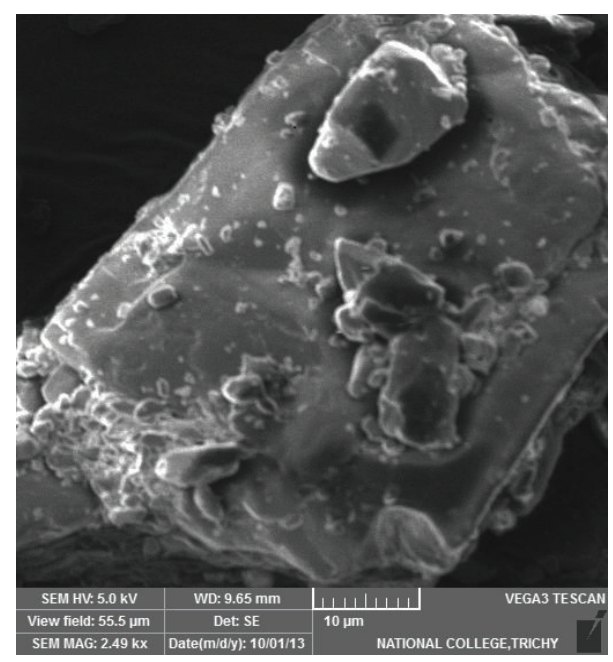

(b)

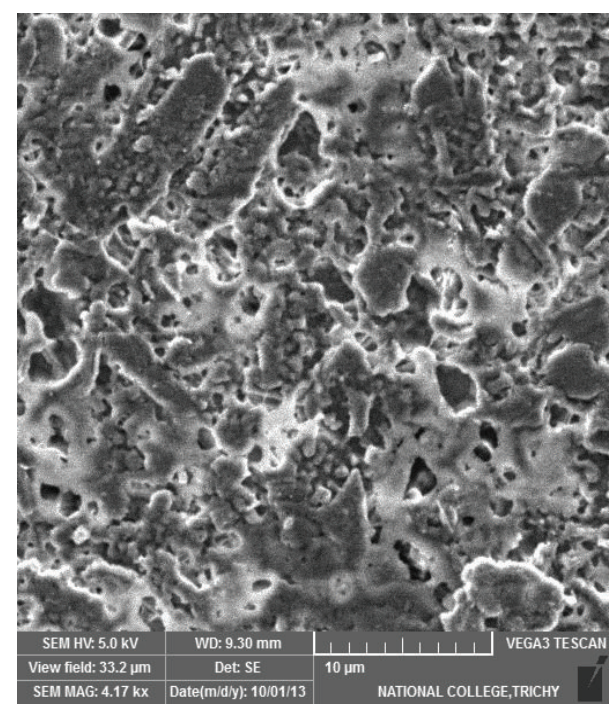

(d)

FIgURE 5: SEM images of (a) etoricoxib, (b) BCD, (c) etoricoxib-BCD complex, and (d) etoricoxib MDF.

strength and desired rapid disintegration which on administration will result in rapid therapeutic action and could be used as an alternate to the commercially available immediate release tablets resulting in improved patient adherence.

\section{Conflict of Interests}

The authors declare that there is no conflict of interests regarding the publication of this paper.

\section{References}

[1] S. Gaisford, A. Verma, M. Saunders, and P. G. Royall, "Monitoring crystallisation of drugs from fast-dissolving oral films with isothermal calorimetry," International Journal of Pharmaceutics, vol. 380, no. 1-2, pp. 105-111, 2009.

[2] http://www.medicines.org.uk/EMC/medicine/8734/SPC/ Arcoxia+30mg.
[3] A. Okumu, M. DiMaso, and R. Löbenberg, "Computer simulations using GastroPlus to justify a biowaiver for etoricoxib solid oral drug products," European Journal of Pharmaceutics and Biopharmaceutics, vol. 72, no. 1, pp. 91-98, 2009.

[4] A. Das, A. K. Nayak, B. Mohanty, and S. Panda, "Solubility and dissolution enhancement of etoricoxib by solid dispersion technique using sugar carriers," ISRN Pharmaceutics, vol. 2011, Article ID 819765, 8 pages, 2011.

[5] Z. Ayenew, V. Puri, L. Kumar, and A. K. Bansal, "Trends in pharmaceutical taste masking technologies: a patent review," Recent Patents on Drug Delivery and Formulation, vol. 3, no. 1, pp. 26-39, 2009.

[6] V. Anand, M. Kataria, V. Kukkar, V. Saharan, and P. K. Choudhury, "The latest trends in the taste assessment of pharmaceuticals," Drug Discovery Today, vol. 12, no. 5-6, pp. 257-265, 2007.

[7] G. Tiwari, R. Tiwari, and K. A. Rai, "Cyclodextrins in delivery systems: applications," Journal of Pharmacy \& Bioallied Sciences, vol. 2, no. 2, pp. 72-79, 2010. 
[8] J. Szejtli and L. Szente, "Elimination of bitter, disgusting tastes of drugs and foods by cyclodextrins," European Journal of Pharmaceutics and Biopharmaceutics, vol. 61, no. 3, pp. 115-125, 2005.

[9] H. M. Patel, B. N. Suhagia, S. A. Shah, I. S. Rathod, and V. K. Parmar, "Preparation and characterization of etoricoxib- $\beta$ cyclodextrin complexes prepared by the kneading method," Acta Pharmaceutica, vol. 57, no. 3, pp. 351-359, 2007.

[10] S. K. Yellanki, S. Jagtap, and R. Masareddy, "Dissofilm: a novel approach for delivery of phenobarbital; design and characterization," Journal of Young Pharmacists, vol. 3, no. 3, pp. 181-188, 2011.

[11] P. Joshi, H. Patel, V. Patel, and R. Panchal, "Formulation development and evaluation of mouth dissolving film of domperidone," Journal of Pharmacy and Bioallied Sciences, vol. 4, no. 5, pp. S108-S109, 2012.

[12] E. M. Janben, R. Schliephacke, A. Breitenbach, and J. Breitkreutz, "Drug-printing by flexographic printing technologya new manufacturing process for orodispersible films," International Journal of Pharmaceutics, vol. 441, no. 1-2, pp. 818-825, 2013.

[13] N. A. Nafee, N. A. Boraie, F. A. Ismail, and L. M. Mortada, "Design and characterization of mucoadhesive buccal patches containing cetylpyridinium chloride," Acta Pharmaceutica, vol. 53, no. 3, pp. 199-212, 2003.

[14] M. J. Akhtar, M. Jacquot, M. Jamshidian, M. Imran, E. ArabTehrany, and S. Desobry, "Fabrication and physicochemical characterization of HPMC films with commercial plant extract: influence of light and film composition," Food Hydrocolloids, vol. 31, no. 2, pp. 420-427, 2013.

[15] Y. Pranoto, C. M. Lee, and H. J. Park, "Characterizations of fish gelatin films added with gellan and $\kappa$-carrageenan," $L W T$-Food Science and Technology, vol. 40, no. 5, pp. 766-774, 2007.

[16] L. Sievens-Figueroa, A. Bhakay, J. I. Jerez-Rozo et al., "Preparation and characterization of hydroxypropyl methyl cellulose films containing stable BCS Class II drug nanoparticles for pharmaceutical applications," International Journal of Pharmaceutics, vol. 423, no. 2, pp. 496-508, 2012.

[17] A. Q. J. Low, J. Parmentier, Y. M. Khong et al., "Effect of type and ratio of solubilising polymer on characteristics of hotmelt extruded orodispersible films," International Journal of Pharmaceutics, vol. 455, no. 1-2, pp. 138-147, 2013.

[18] H. F. Brinson and L. C. Brinson, "Polymer engineering science and viscoelasticity: an introduction," in Stress and Strain Analysis and Measurement, pp. 15-54, Springer Science + Business Media, New York, NY, USA, 2008.

[19] M. Haque, S. Nasrin, M. M. Monir, M. M. Rahman, and S. Chowdhury, "Method development and validation of RP-HPLC method of etoricoxib in bulk and its tablet dosage forms," American Journal of PharmTech Research, vol. 2, no. 6, pp. 275283, 2012.

[20] S. Topalli, T. G. Chandrasekhar, and M. M. Annapurna, "Validated RP-HPLC method for the assay of etoricoxib (a nonsteroidal anti-inflammatory drug) in pharmaceutical dosage forms," E-Journal of Chemistry, vol. 9, no. 2, pp. 832-838, 2012.

[21] S. V. R. Reddy, S. Dondeti, R. Manavalan, and J. Sreekanth, "Palatability evaluation study of oral disintegrating tablets by human volunteers," International Journal of Pharma Sciences and Research, vol. 1, no. 8, pp. 326-346, 2010.

[22] M. Goyal, M. Ghosh, B. P. Nagori, and D. Sasmal, "Analgesic and anti-inflammatory studies of cyclopeptide alkaloid fraction of leaves of Ziziyphus nummularia," Saudi Journal of Biological Sciences, vol. 20, no. 4, pp. 365-371, 2013.

[23] S. K. Kulkarni, Hand Book of Experimental Pharmacology, Vallabh Prakashan, New Delhi, India, 3rd edition, 2009.

[24] D. Mishra, G. Ghosh, P. S. Kumar, and P. K. Panda, "An experimental study of analgesic activity of selective COX-2 inhibitor with conventional NSAIDs," Asian Journal of Pharmaceutical and Clinical Research, vol. 4, no. 1, pp. 78-81, 2011.

[25] S.-Y. Lin, C.-H. Hsu, and M.-T. Sheu, "Curve-fitting FTIR studies of loratadine/hydroxypropyl- $\beta$-cyclodextrin inclusion complex induced by co-grinding process," Journal of Pharmaceutical and Biomedical Analysis, vol. 53, no. 3, pp. 799-803, 2010.

[26] U. S. Department of Health and Human Services, Food, and Drug Administration, Guidance for Industry, Orally Disintegrating Tablets, U. S. Department of Health and Human Services, FDA, 2008.

[27] S. Shimpi, K. Mahadik, K. Takada, and A. Paradkar, "Application of polyglycolized glycerides in protection of amorphous form of etoricoxib during compression," Chemical and Pharmaceutical Bulletin, vol. 55, no. 10, pp. 1448-1451, 2007.

[28] A. H. Al-Marzouqi, H. M. Elwy, I. Shehadi, and A. Adem, "Physicochemical properties of antifungal drug-cyclodextrin complexes prepared by supercritical carbon dioxide and by conventional techniques," Journal of Pharmaceutical and Biomedical Analysis, vol. 49, no. 2, pp. 227-233, 2009.

[29] Z. Aigner, O. Berkesi, G. Farkas, and P. Szabó-Révész, “DSC, Xray and FTIR studies of a gemfibrozil/dimethyl- $\beta$-cyclodextrin inclusion complex produced by co-grinding," Journal of Pharmaceutical and Biomedical Analysis, vol. 57, no. 1, pp. 62-67, 2012. 

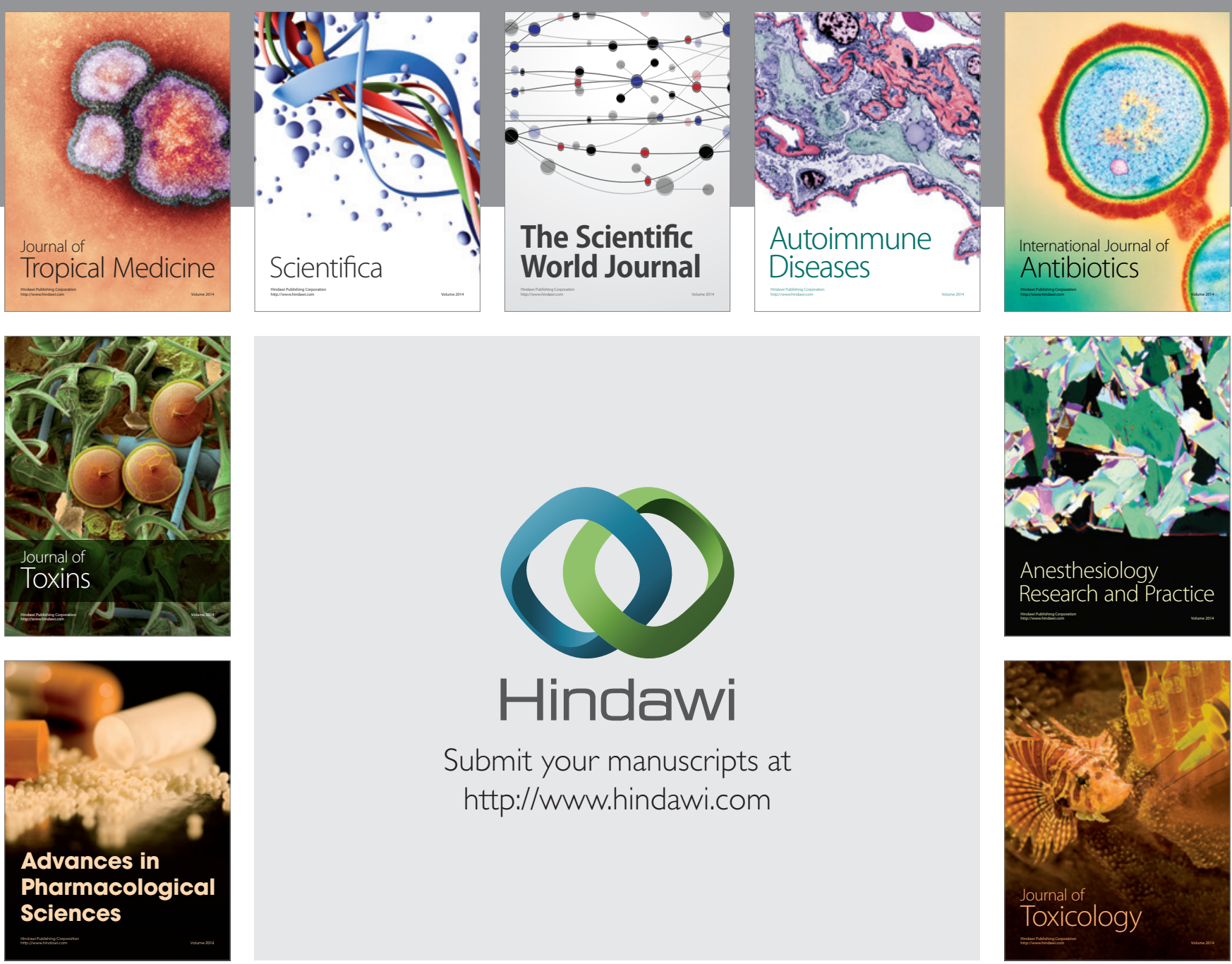

\section{Hindawi}

Submit your manuscripts at

http://www.hindawi.com
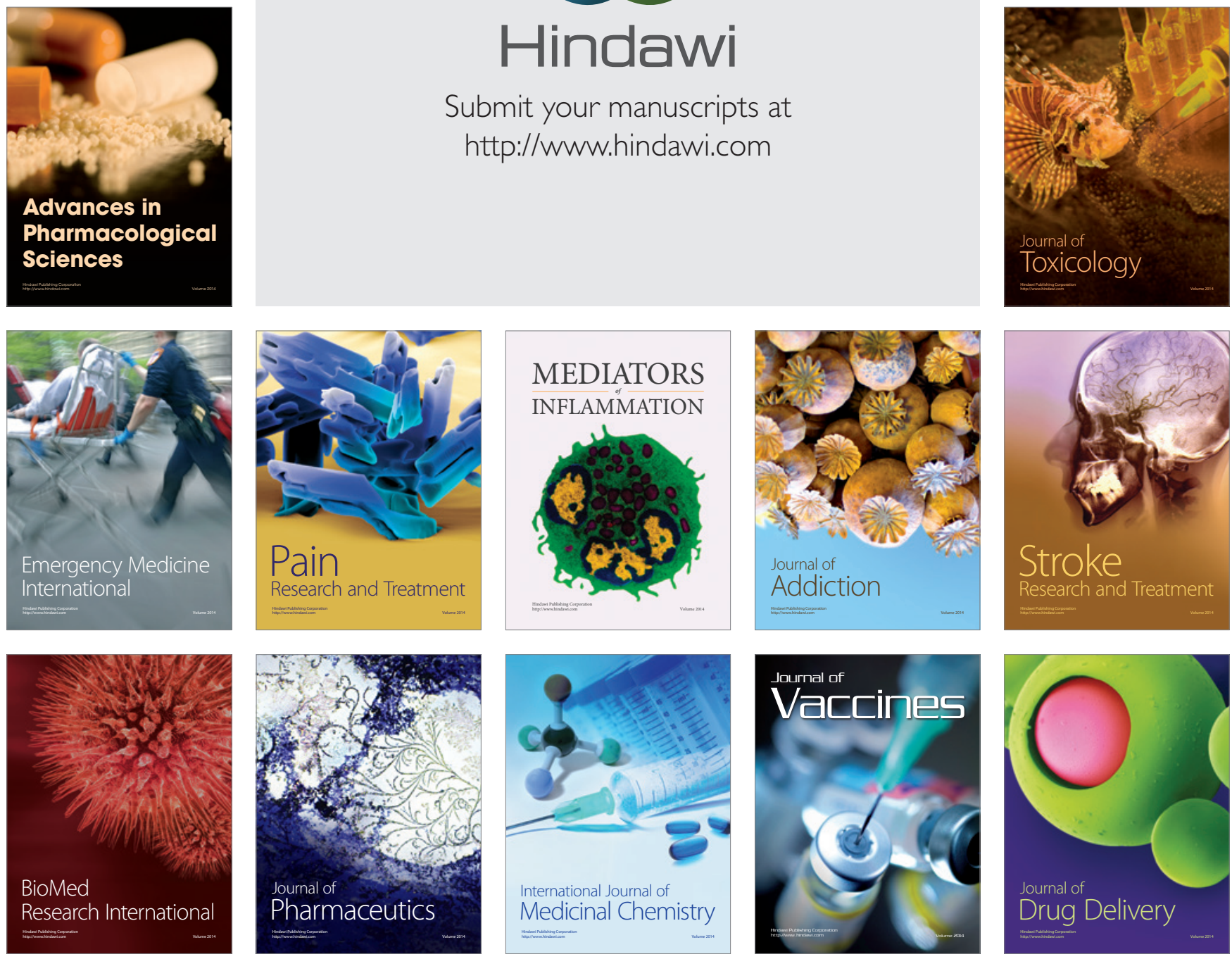